Supporting Information

\title{
DFT Based Theoretical Study about the Contributions of Fluorine to Nonlinear Optical Properties in Borate Fluoride Crystals
}

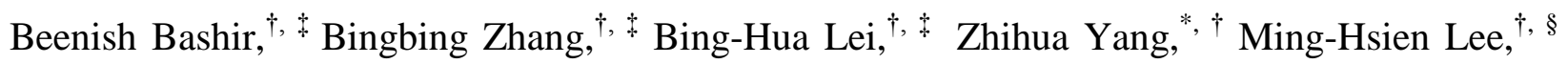
and Shilie $\operatorname{Pan}^{*}+\dagger$

${ }^{\dagger}$ Key Laboratory of Functional Materials and Devices for Special Environments, Xinjiang Technical Institute of Physics \& Chemistry, Chinese Academy of Sciences, Xinjiang Key Laboratory of Electronic Information Materials and Devices, 40-1 South Beijing Road, Urumqi 830011, China

${ }^{+}$University of Chinese Academy of Sciences, Beijing 100049, China

${ }^{\S}$ Department of Physics, Tamkang University, New Taipei City 25137, Taiwan

*To whom corresponding should be addressed.

E- mail: zhyang@ms.xjb.ac.cn (Zhihua Yang), slpan@ms.xjb.ac.cn (Shilie Pan), Tel (+86) -9913810816; Fax: (+86)991-3838957.

Table S1. The Crstal description of $\mathrm{Sr}_{3} \mathrm{~B}_{6} \mathrm{O}_{11} \mathrm{~F}_{2}, \mathrm{Ba}_{3} \mathrm{~B}_{6} \mathrm{O}_{11} \mathrm{~F}_{2}, \mathrm{Ba}_{4} \mathrm{~B}_{11} \mathrm{O}_{20} \mathrm{~F}$, and $\mathrm{Sr}_{4} \mathrm{~B}_{10} \mathrm{O}_{18}(\mathrm{OH})_{2} \cdot 2 \mathrm{H}_{2} \mathrm{O}$.

Table S2. Calculated energy band gaps by LDA and GGA functionals and experiment band gap of studied compounds. 
Table S3. The calcualted SHG tensors and exprimental powder SHG (PSHG) intesnities of title compounds. Whereas $\mathrm{d}_{36}(\mathrm{KDP})=0.39 \mathrm{pm} / \mathrm{V}$.

Figure S1. The 3D framework of (a) $\mathrm{Ba}_{4} \mathrm{~B}_{11} \mathrm{O}_{20} \mathrm{~F}$ (b) $\mathrm{Ba}_{3} \mathrm{~B}_{6} \mathrm{O}_{11} \mathrm{~F}_{2}$ compounds.

Figure S2. Calculated band structures of (a) $\mathrm{Sr}_{3} \mathrm{~B}_{6} \mathrm{O}_{11} \mathrm{~F}_{2}$, (b) $\mathrm{Ba}_{3} \mathrm{~B}_{6} \mathrm{O}_{11} \mathrm{~F}_{2}$, (c) $\mathrm{Ba}_{4} \mathrm{~B}_{11} \mathrm{O}_{20} \mathrm{~F}$, (d) $\mathrm{Ca}_{5}\left(\mathrm{BO}_{3}\right)_{3} \mathrm{~F}$, and (e) $\mathrm{Sr}_{4} \mathrm{~B}_{10} \mathrm{O}_{18}(\mathrm{OH})_{2} \cdot 2 \mathrm{H}_{2} \mathrm{O}$.

Figure S3. The PDOS (top) and band-resolved $\chi^{(2)}$ (bottom) of (a) $\mathrm{Ba}_{3} \mathrm{~B}_{6} \mathrm{O}_{11} \mathrm{~F}_{2}$, (b) $\mathrm{Ba}_{4} \mathrm{~B}_{11} \mathrm{O}_{20} \mathrm{~F}$. The PDOS and band-resolved $\chi^{(2)}$ demonstrate that region-I in VBs and region-II in CBs makes the significant contribution to the SHG effect.

Figure S4. The PDOS (top) and band-resolved $\chi^{(2)}$ (bottom) of $\mathrm{Ca}_{5}\left(\mathrm{BO}_{3}\right)_{3} \mathrm{~F}$. The PDOS and band-resolved $\chi^{(2)}$ demonstrate that region-I in VBs and region-II in CBs makes the significant contribution to the SHG effect.

Figure S5. Some respective orbitals of $\mathrm{Sr}_{3} \mathrm{~B}_{6} \mathrm{O}_{11} \mathrm{~F}_{2}$.

Figure S6. The SHG-density of occupied states and unoccupied states of $\mathrm{Ba}_{4} \mathrm{~B}_{11} \mathrm{O}_{20} \mathrm{~F}$ in $\mathrm{VE}$ process.

Figure S7. The SHG-density of occupied states and unoccupied states of $\mathrm{Ca}_{5}\left(\mathrm{BO}_{3}\right)_{3} \mathrm{~F}$ in VE process. 
Table S1. The Crstal description of $\mathrm{Sr}_{3} \mathrm{~B}_{6} \mathrm{O}_{11} \mathrm{~F}_{2}{ }^{[1]}, \mathrm{Ba}_{3} \mathrm{~B}_{6} \mathrm{O}_{11} \mathrm{~F}_{2}{ }^{[2]}, \mathrm{Ba}_{4} \mathrm{~B}_{11} \mathrm{O}_{20} \mathrm{~F}^{[3]}$ and $\mathrm{Sr}_{4} \mathrm{~B}_{10} \mathrm{O}_{18}(\mathrm{OH})_{2} .2 \mathrm{H}_{2} \mathrm{O}^{[4]}$.

\begin{tabular}{|c|c|c|c|}
\hline Crystal formula & Space group & Crystal system & Cell parameters \\
\hline \multirow[t]{4}{*}{$\mathrm{Sr}_{3} \mathrm{~B}_{6} \mathrm{O}_{11} \mathrm{~F}_{2}$} & $P 2_{1}$ & Monoclinic & $a=6.3847(6) \AA$ \\
\hline & & & $\mathrm{b}=8.2493(9) \AA$ \\
\hline & & & $\mathrm{c}=9.3867(9) \AA$ \\
\hline & & & $\beta=101.680(5)^{\circ}$ \\
\hline \multirow[t]{4}{*}{$\mathrm{Ba}_{3} \mathrm{~B}_{6} \mathrm{O}_{11} \mathrm{~F}_{2}$} & $P 2_{1}$ & Monoclinic & $a=6.5672(4) \AA$ \\
\hline & & & $\mathrm{b}=8.5035(6) \AA$ \\
\hline & & & $c=9.6637(6) \AA$ \\
\hline & & & $\beta=101.351(4)^{\circ}$ \\
\hline \multirow[t]{3}{*}{$\mathrm{Ba}_{4} \mathrm{~B}_{11} \mathrm{O}_{20} \mathrm{~F}$} & $\mathrm{Cmc}_{1}$ & Orthorhombic & $a=18.8112(15) \AA$ \\
\hline & & & $b=10.7151(6) \AA$ \\
\hline & & & $c=8.6031(5) \AA$ \\
\hline \multirow[t]{6}{*}{$\mathrm{Sr}_{4} \mathrm{~B}_{10} \mathrm{O}_{18}(\mathrm{OH})_{2} \cdot 2 \mathrm{H}_{2} \mathrm{O}$} & $P 1$ & Triclinic & $a=6.4991(6) \AA$ \\
\hline & & & $b=6.5059(6) \AA$ \\
\hline & & & $\mathrm{c}=11.3175(11) \AA$ \\
\hline & & & $\alpha=88.458(6)^{\circ}$ \\
\hline & & & $\beta=86.019(5)^{\circ}$ \\
\hline & & & $\gamma=61.351(5)^{\circ}$ \\
\hline
\end{tabular}


Table S2. Calculated energy band gaps by LDA and GGA functionals and experiment band gap of studied compounds.

\begin{tabular}{cccc}
\hline Band gap $(\mathrm{eV})$ & & & \\
\hline Compounds & LDA & GGA & Experiment \\
\hline $\mathrm{Sr}_{3} \mathrm{~B}_{6} \mathrm{O}_{11} \mathrm{~F}_{2}$ & 5.33 & 5.66 & 5.84 \\
$\mathrm{Ba}_{3} \mathrm{~B}_{6} \mathrm{O}_{11} \mathrm{~F}_{2}$ & 5.11 & 5.39 & 5.84 \\
$\mathrm{Ba}_{4} \mathrm{~B}_{11} \mathrm{O}_{20} \mathrm{~F}$ & 4.59 & 4.85 & 7.08 \\
$\mathrm{Ca}_{5}\left(\mathrm{BO}_{3}\right)_{3} \mathrm{~F}$ & 4.07 & 4.36 & 6.33 \\
$\mathrm{Sr}_{4} \mathrm{~B}_{10} \mathrm{O}_{18}(\mathrm{OH})_{2} \cdot 2 \mathrm{H}_{2} \mathrm{O}$ & 5.01 & 5.32 & 6.08 \\
\hline
\end{tabular}

Table S3. The calcualted SHG tensors and exprimental powder SHG (PSHG) intesnities of title compounds. Whereas $\mathrm{d}_{36}(\mathrm{KDP})=0.39 \mathrm{pm} / \mathrm{V}$.

\begin{tabular}{ccccc}
\hline Compounds & $\begin{array}{c}\text { Calculated SHG } \\
\text { tensors }(\mathrm{pm} / \mathrm{V})\end{array}$ & $\mathrm{VE}$ & $\mathrm{VH}$ & PSHG intensity \\
\hline $\mathrm{Sr}_{3} \mathrm{~B}_{6} \mathrm{O}_{11} \mathrm{~F}_{2}$ & $\mathrm{~d}_{21}=-1.777$ & -1.2665 & -0.5107 & $2.5 \mathrm{KDP}^{[1]}$ \\
& $\mathrm{d}_{36}=-0.609$ & -0.5463 & -0.1437 & \\
& $\mathrm{~d}_{22}=1.165$ & 1.1717 & 0.6134 & \\
$\mathrm{Ca}_{5}\left(\mathrm{BO}_{3}\right)_{3} \mathrm{~F}^{[5]}$ & $\mathrm{d}_{11}=0.609$ & 1.1507 & 0.0684 & $2 \mathrm{KDP}^{[5]}$ \\
& $\mathrm{d}_{12}=-0.839$ & -1.3185 & -0.3602 & \\
& $\mathrm{~d}_{13}=-0.069$ & -0.0905 & -0.0481 & \\
& $\mathrm{~d}_{15}=0.487$ & 0.8119 & 0.1621 & \\
& $\mathrm{~d}_{24}=-0.479$ & -0.6918 & -0.2675 & \\
& $\mathrm{~d}_{33}=-0.029$ & -0.1172 & -0.0572 &
\end{tabular}




$\begin{array}{ccccc}\mathrm{Sr}_{4} \mathrm{~B}_{10} \mathrm{O}_{18}(\mathrm{OH})_{2} \cdot 2 \mathrm{H}_{2} \mathrm{O} & \mathrm{d}_{11}=0.846 & 1.0146 & 0.6787 & 2 \mathrm{KDP}^{[4]} \\ & \mathrm{d}_{22}=1.023 & 1.6531 & 0.3931 \\ & \mathrm{~d}_{33}=-0.933 & -1.7173 & -0.6957\end{array}$

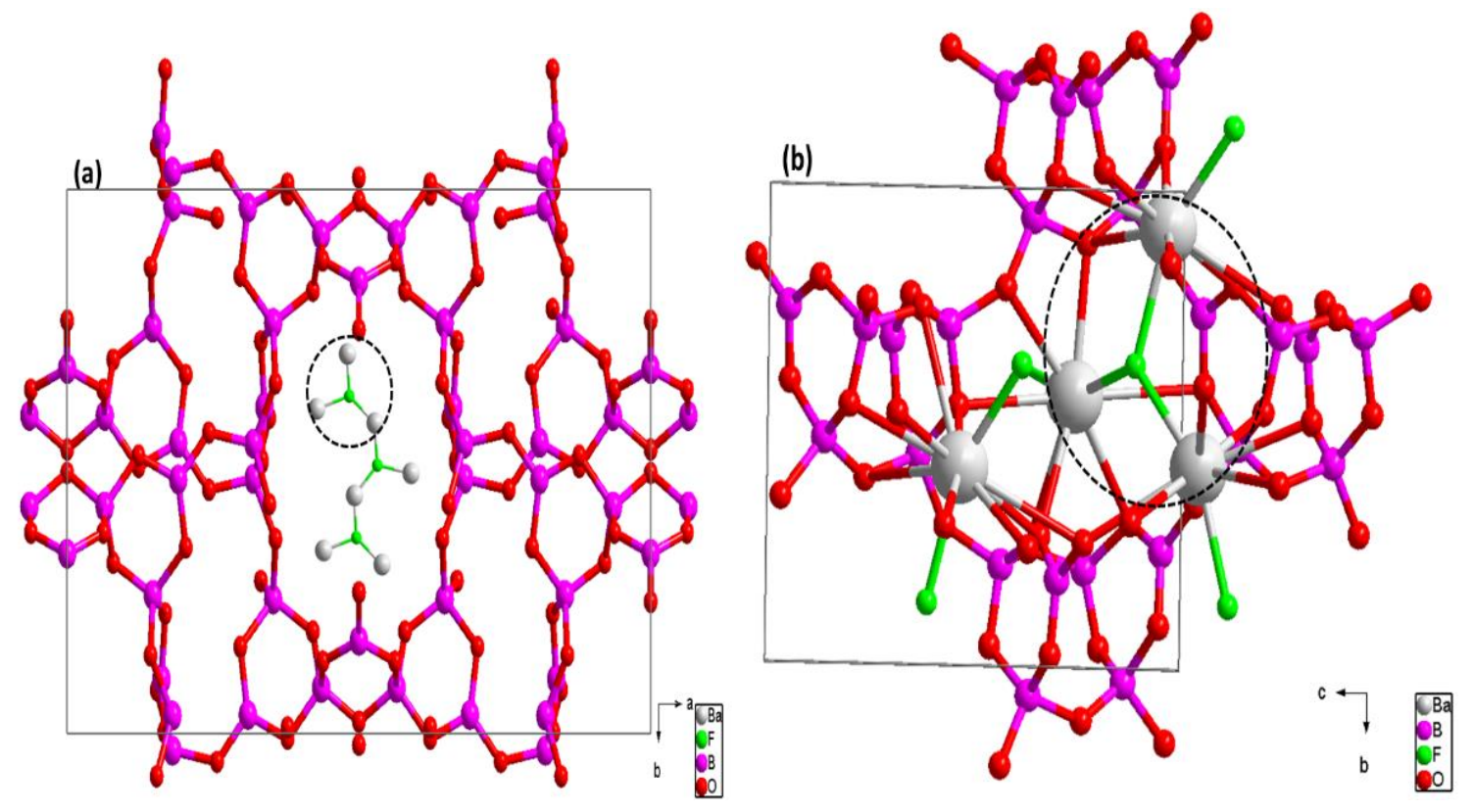

Figure S1. The 3D framework of (a) $\mathrm{Ba}_{4} \mathrm{~B}_{11} \mathrm{O}_{20} \mathrm{~F}$ (b) $\mathrm{Ba}_{3} \mathrm{~B}_{6} \mathrm{O}_{11} \mathrm{~F}_{2}$ compounds. 
(a)

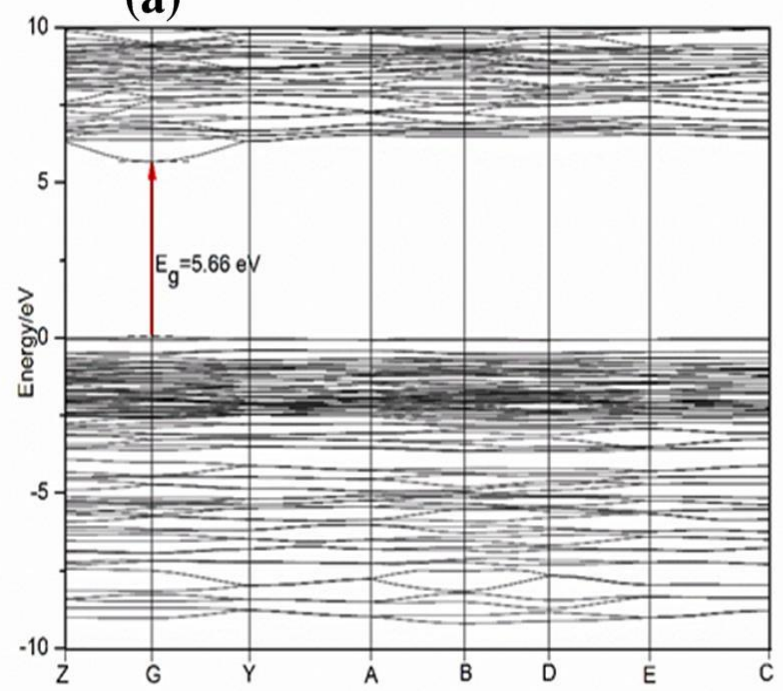

(c)

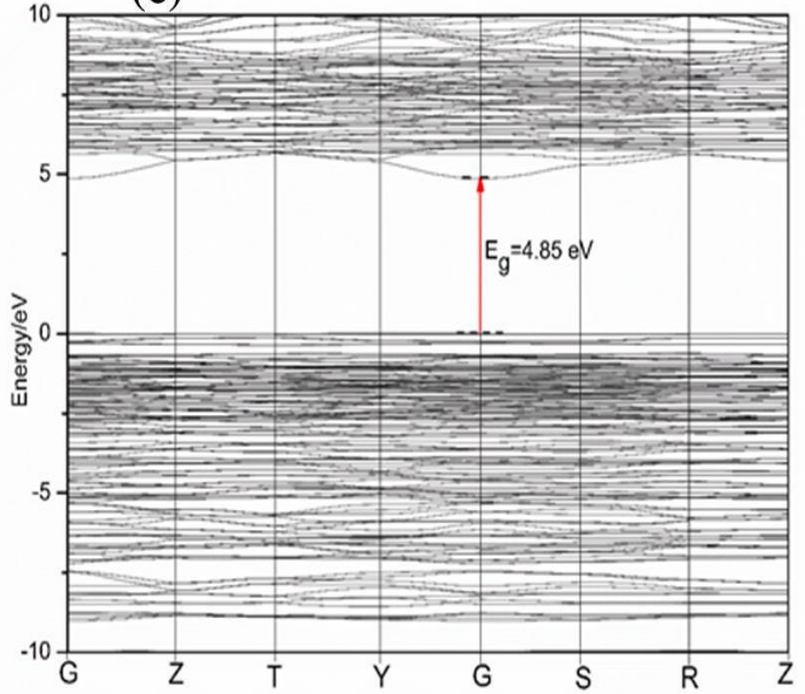

(e)

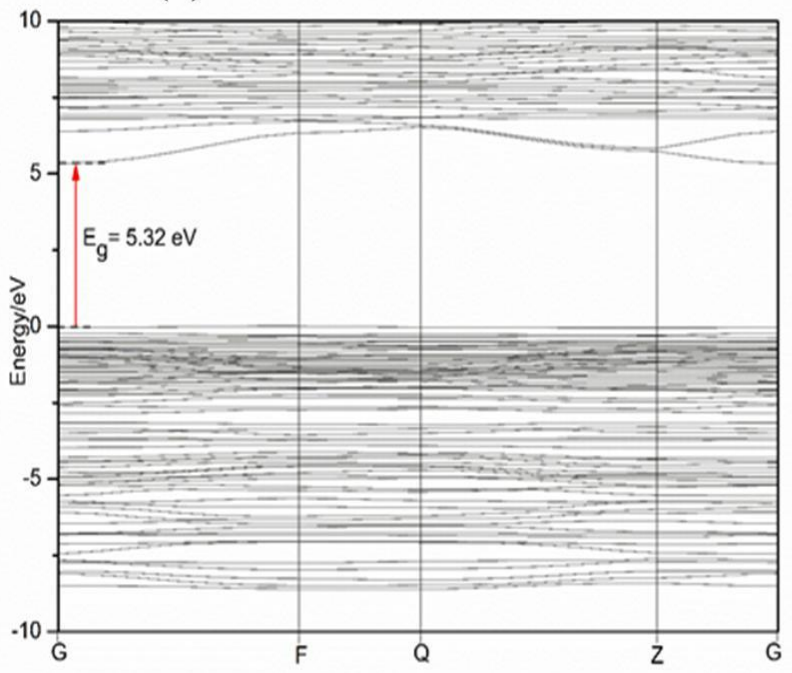

(b)

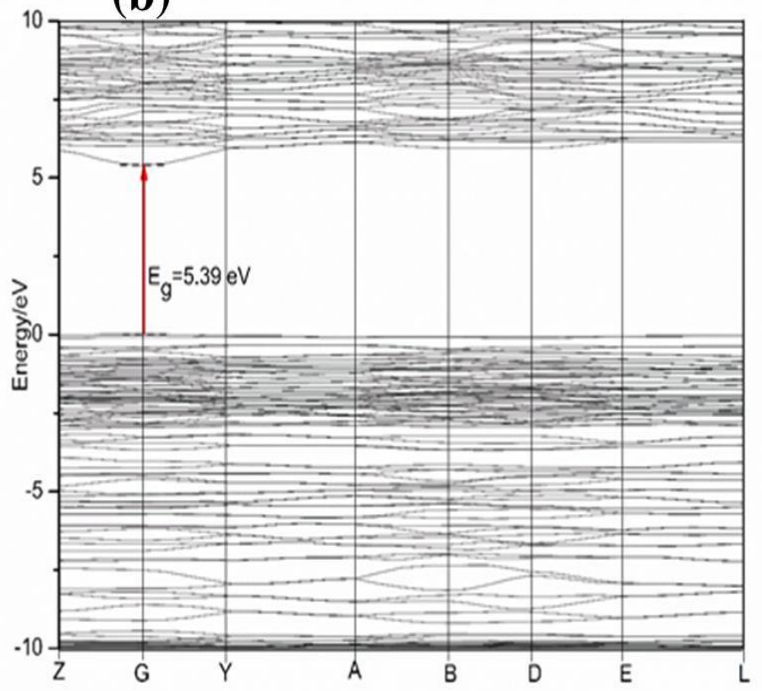

(d)

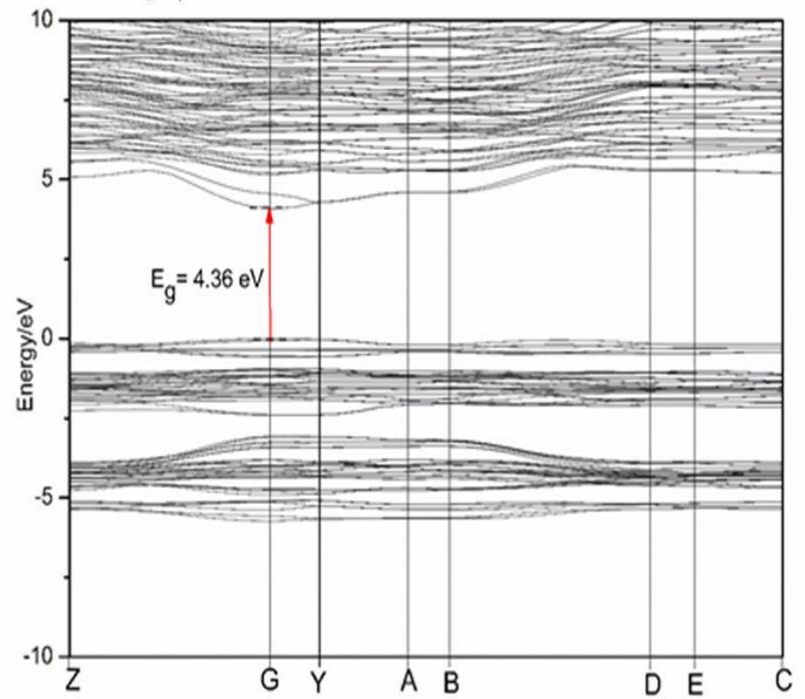


Figure S2. Calculated band structures of (a) $\mathrm{Sr}_{3} \mathrm{~B}_{6} \mathrm{O}_{11} \mathrm{~F}_{2}$, (b) $\mathrm{Ba}_{3} \mathrm{~B}_{6} \mathrm{O}_{11} \mathrm{~F}_{2}$, (c) $\mathrm{Ba}_{4} \mathrm{~B}_{11} \mathrm{O}_{20} \mathrm{~F}$, (d) $\mathrm{Ca}_{5}\left(\mathrm{BO}_{3}\right)_{3} \mathrm{~F}$, and (e) $\mathrm{Sr}_{4} \mathrm{~B}_{10} \mathrm{O}_{18}(\mathrm{OH})_{2} \cdot 2 \mathrm{H}_{2} \mathrm{O}$.
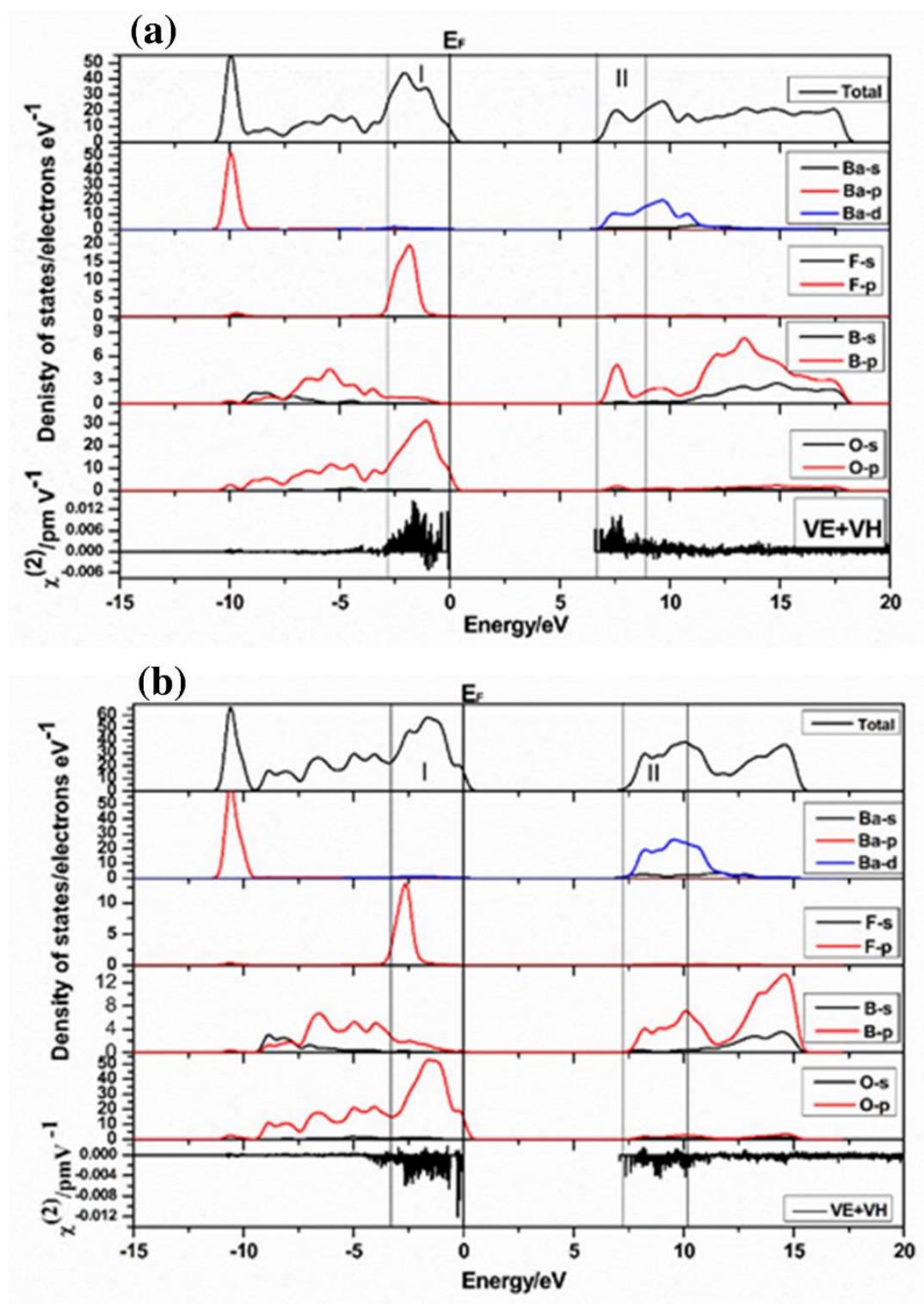

Figure S3. The PDOS (top) and band-resolved $\chi^{(2)}$ (bottom) of (a) $\mathrm{Ba}_{3} \mathrm{~B}_{6} \mathrm{O}_{11} \mathrm{~F}_{2}$, and (b) $\mathrm{Ba}_{4} \mathrm{~B}_{11} \mathrm{O}_{20} \mathrm{~F}$. The PDOS and band-resolved $\chi^{(2)}$ demonstrate that region-I in VBs and region-II in CBs makes the significant contribution to the SHG effect. 


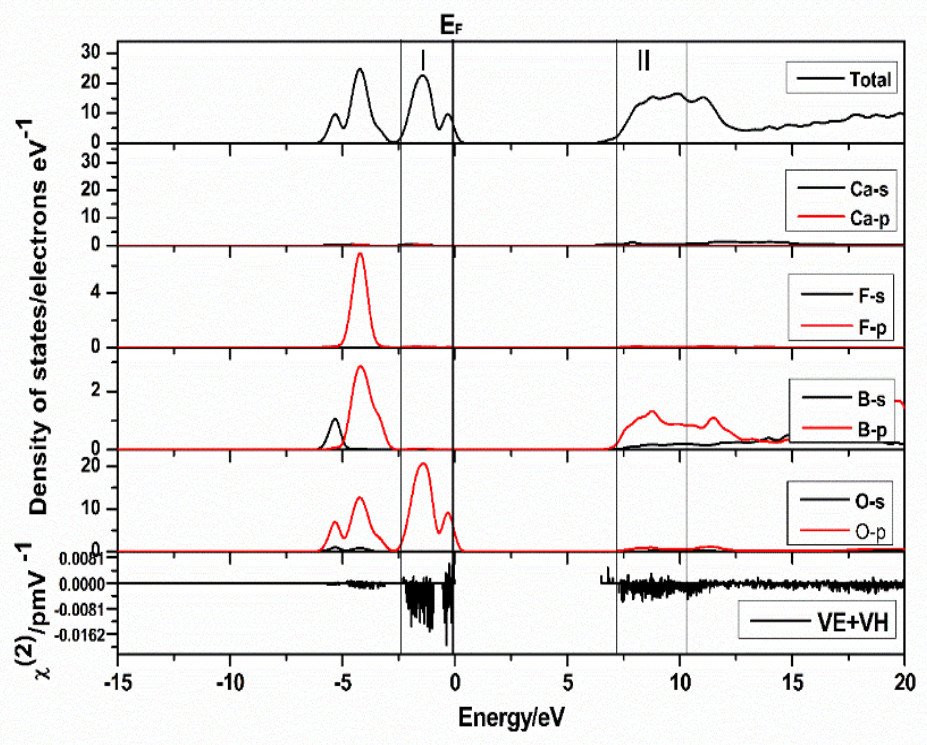

Figure S4. The PDOS (top) and band-resolved $\chi^{(2)}$ (bottom) of $\mathrm{Ca}_{5}\left(\mathrm{BO}_{3}\right)_{3} \mathrm{~F}$. The PDOS and band-resolved $\chi^{(2)}$ demonstrate that region-I in VBs and region-II in CBs makes the significant contribution to the SHG effect. 

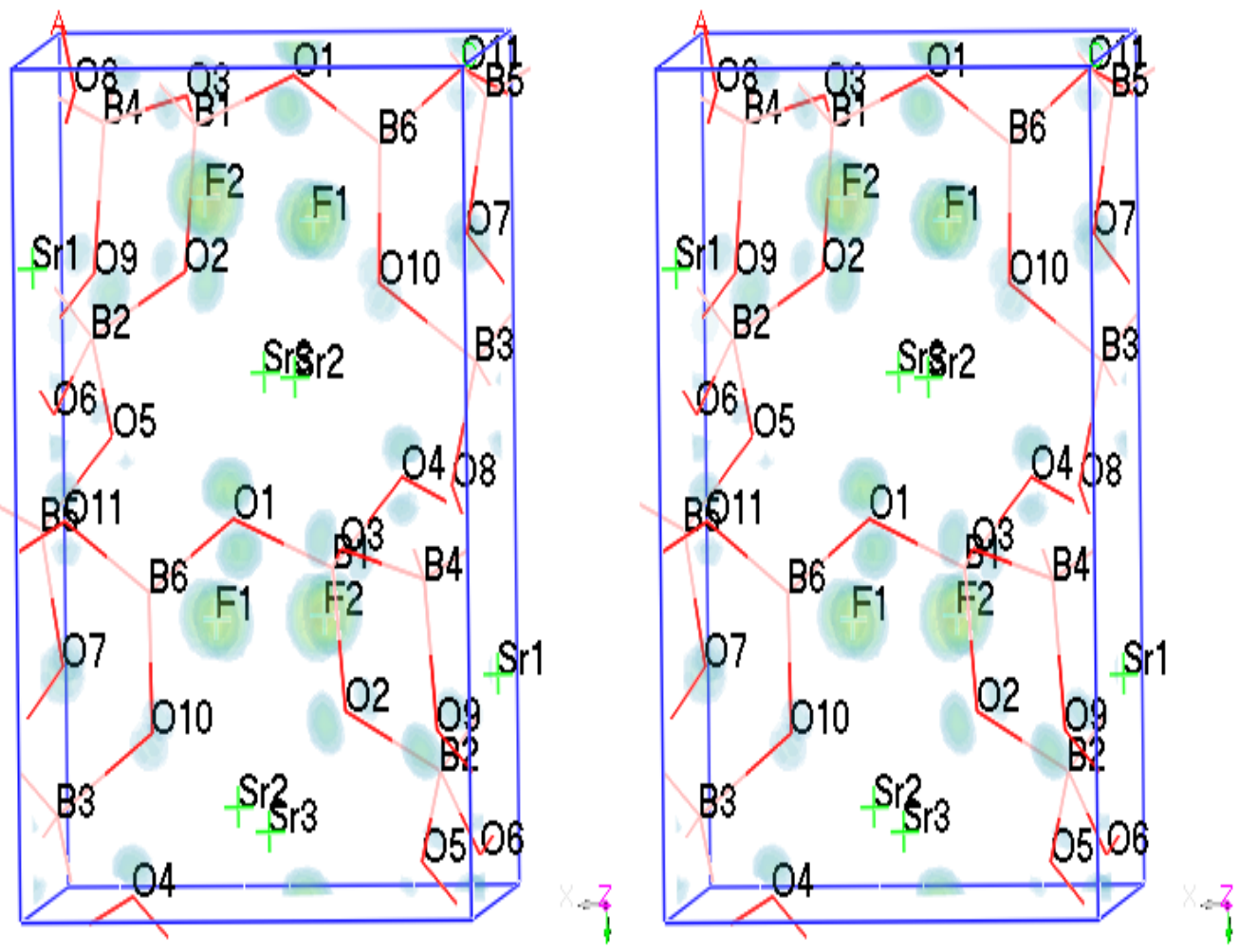

(a) Orbital 108

(b) Orbital 112
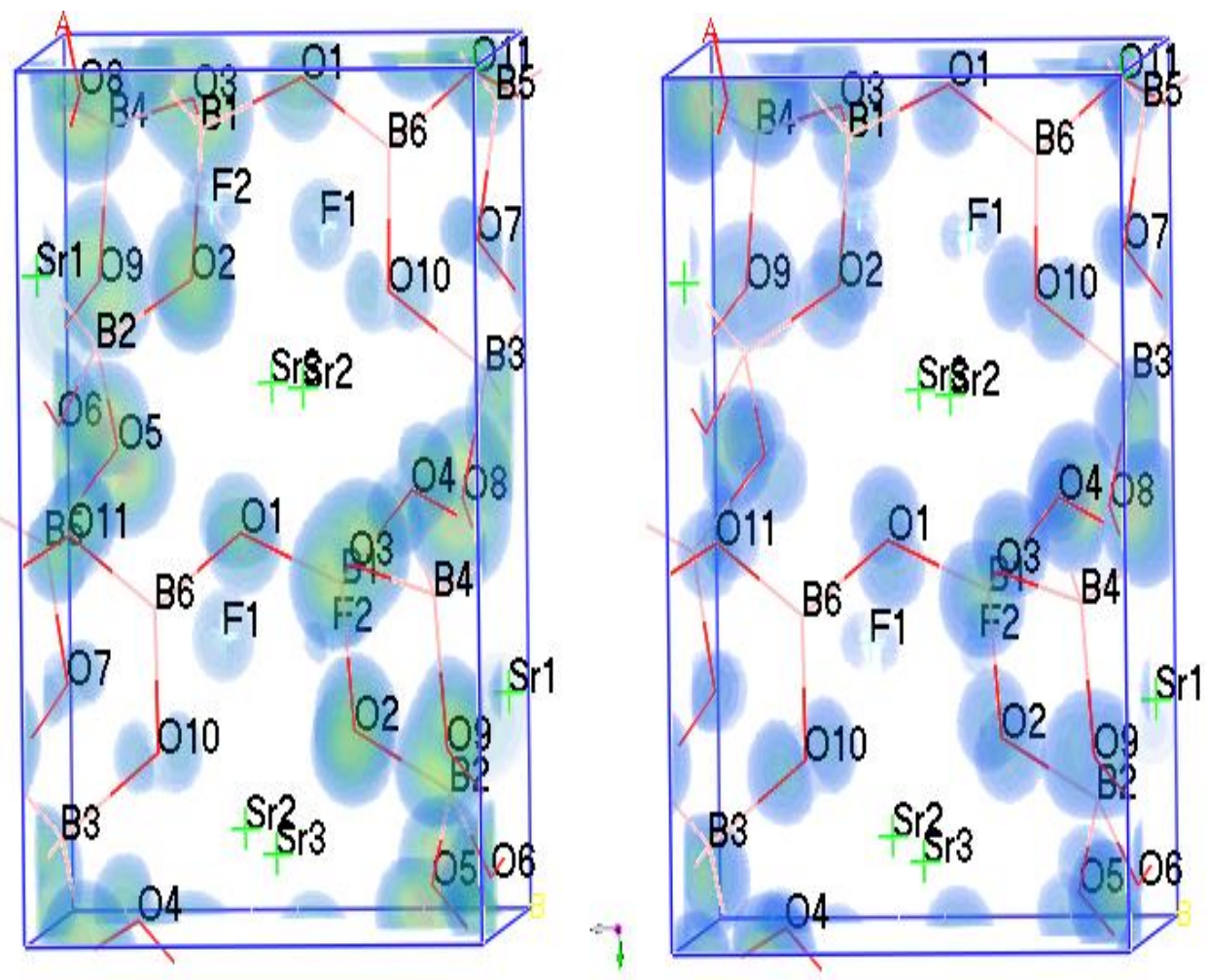
(c) Orbital 120

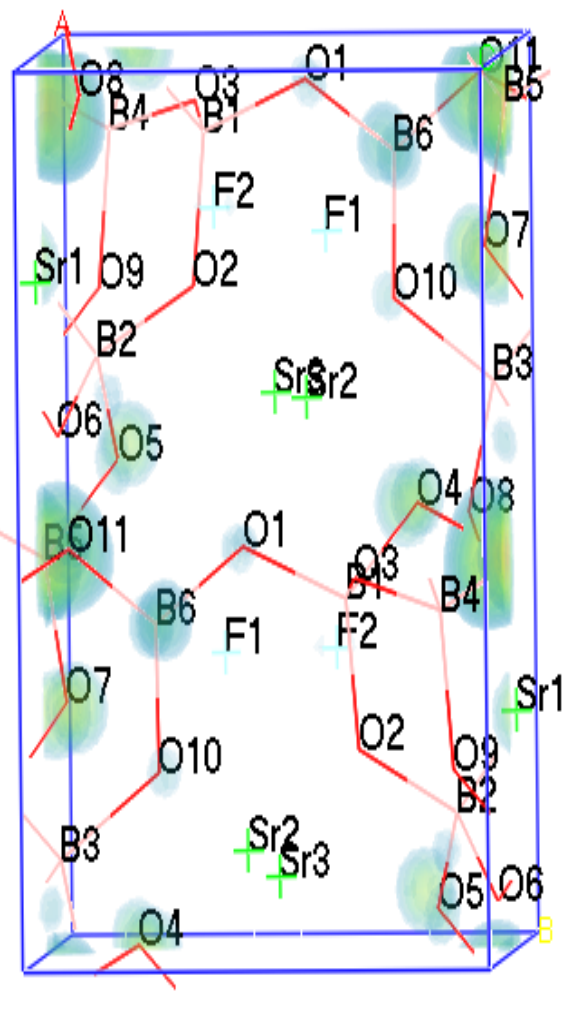

(e) Orbital 129 (LUMO) (d) Orbital 128 (HOMO)

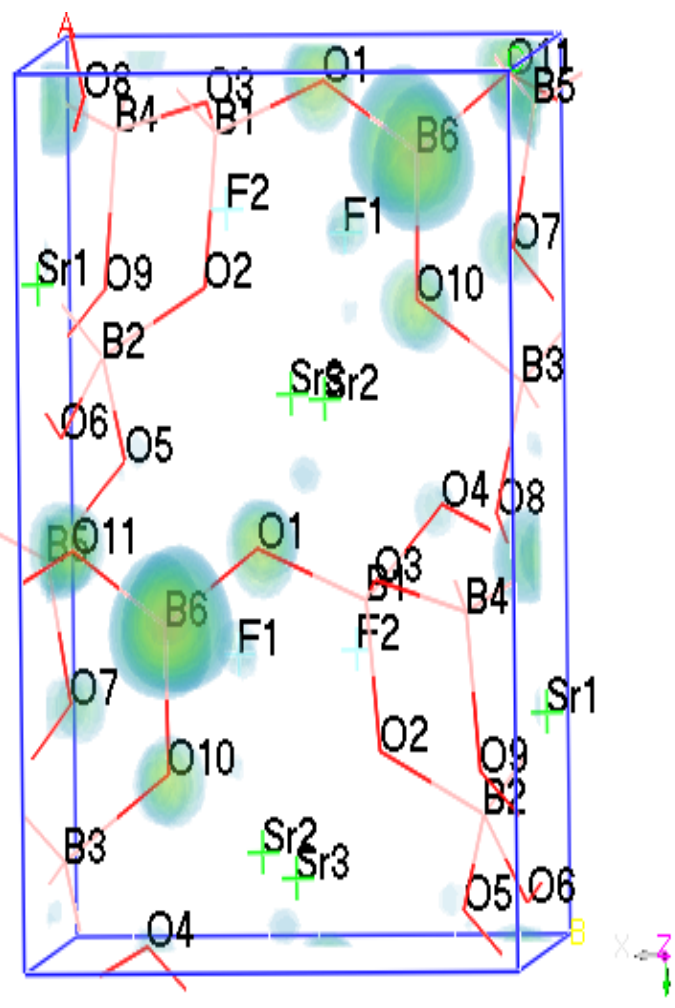

(f) Orbital 134 


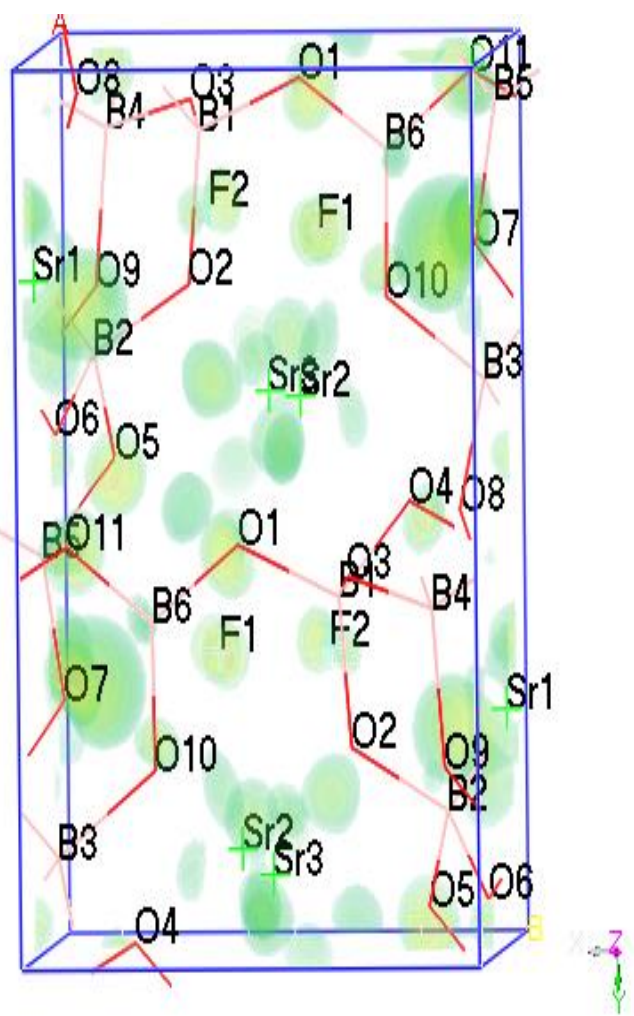

(g) Orbital 140

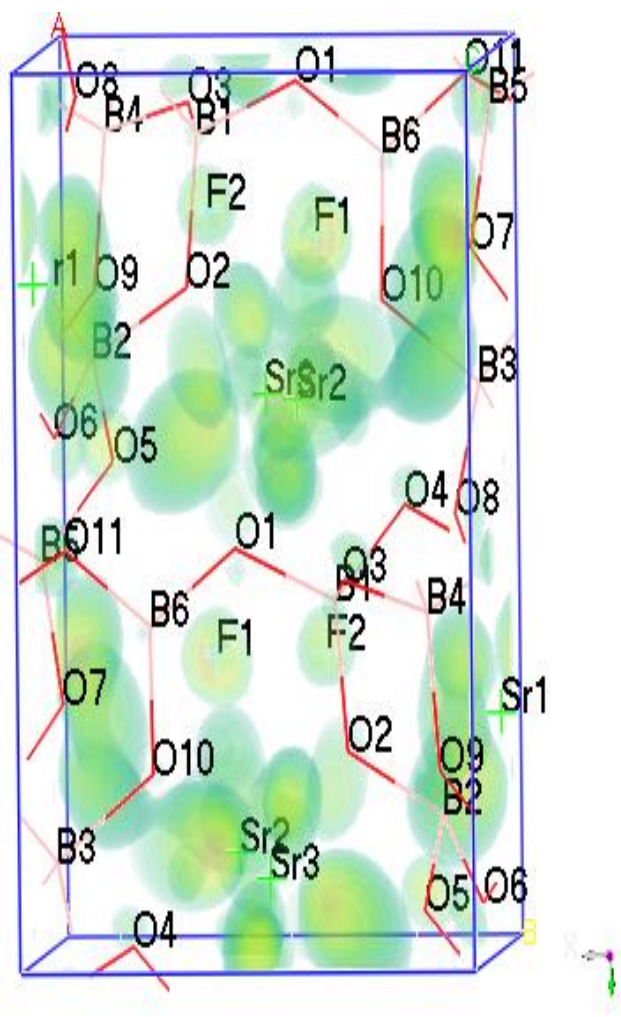

(h) Orbital 146

Figure S5. Some respective orbitals of $\mathrm{Sr}_{3} \mathrm{~B}_{6} \mathrm{O}_{11} \mathrm{~F}_{2}$.

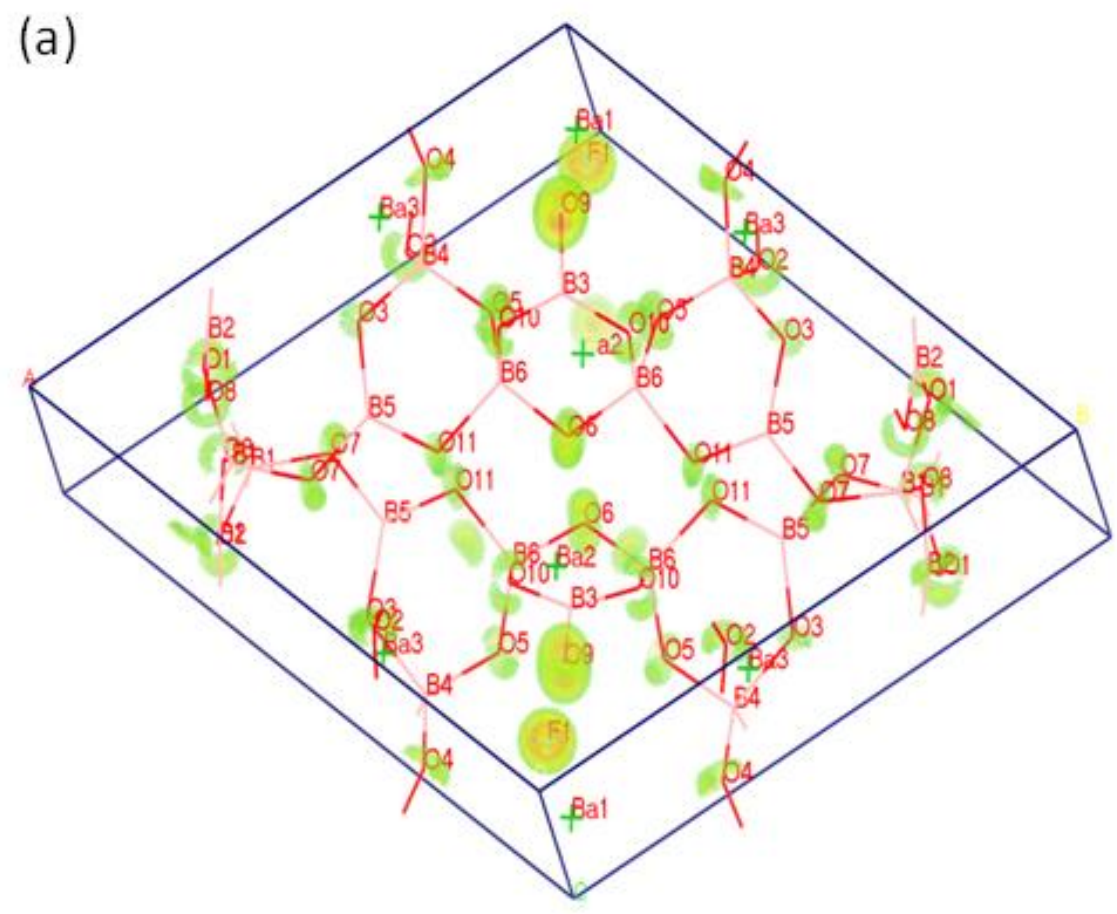

VE occupied

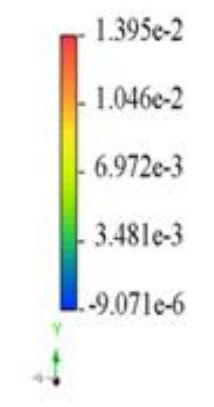


(b)

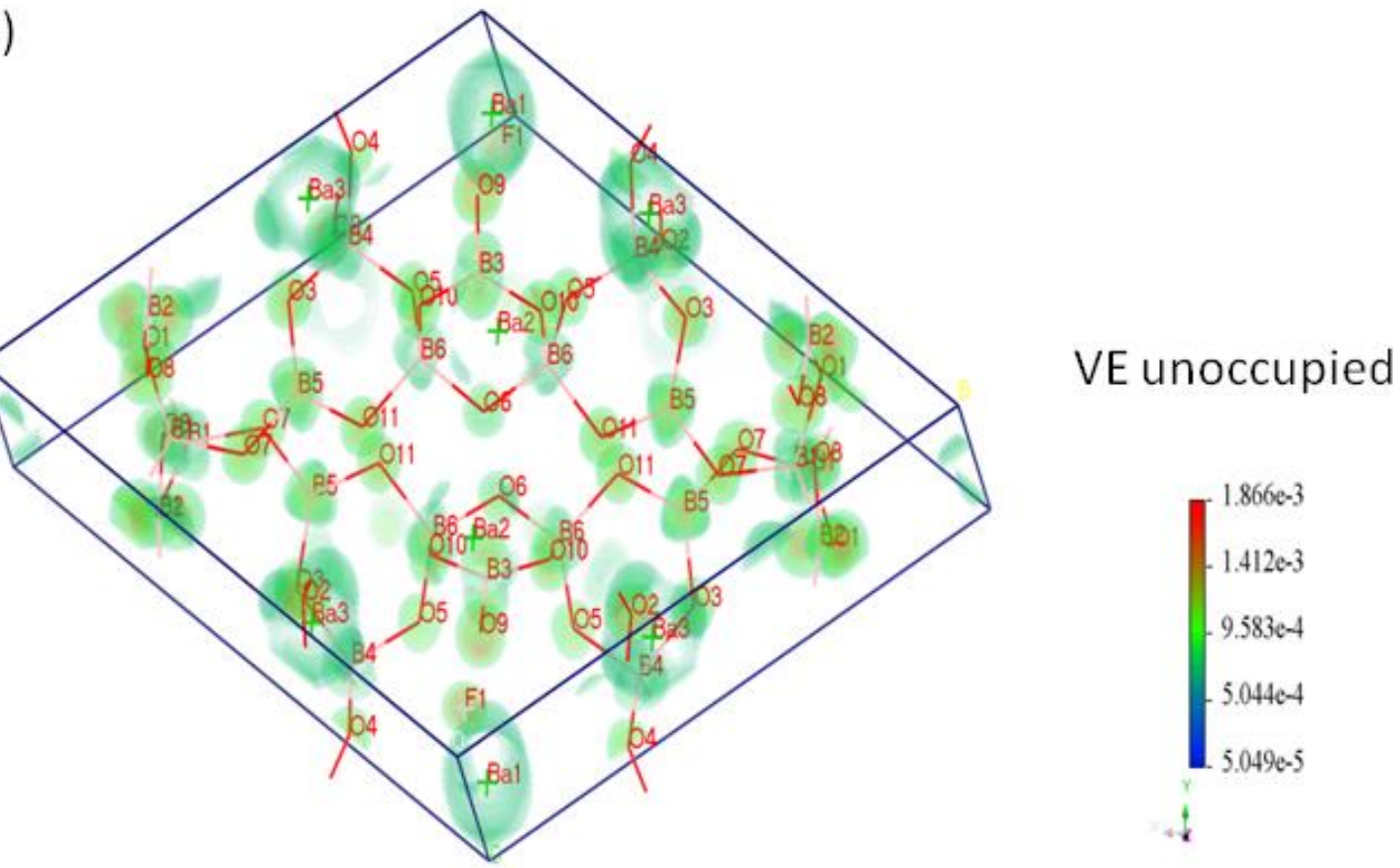

Figure S6. The SHG-density of occupied states and unoccupied states of $\mathrm{Ba}_{4} \mathrm{~B}_{11} \mathrm{O}_{20} \mathrm{~F}$ in VE process. 
(a)

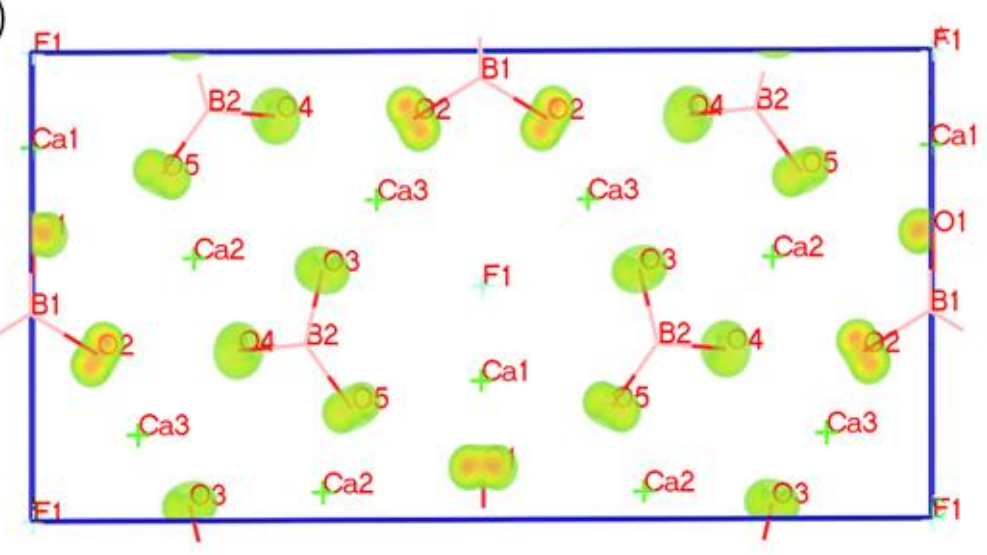

VE occupied

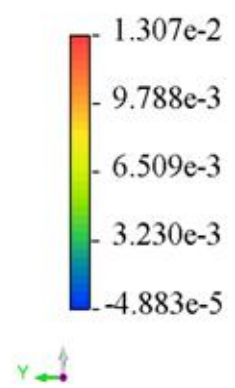

(b)

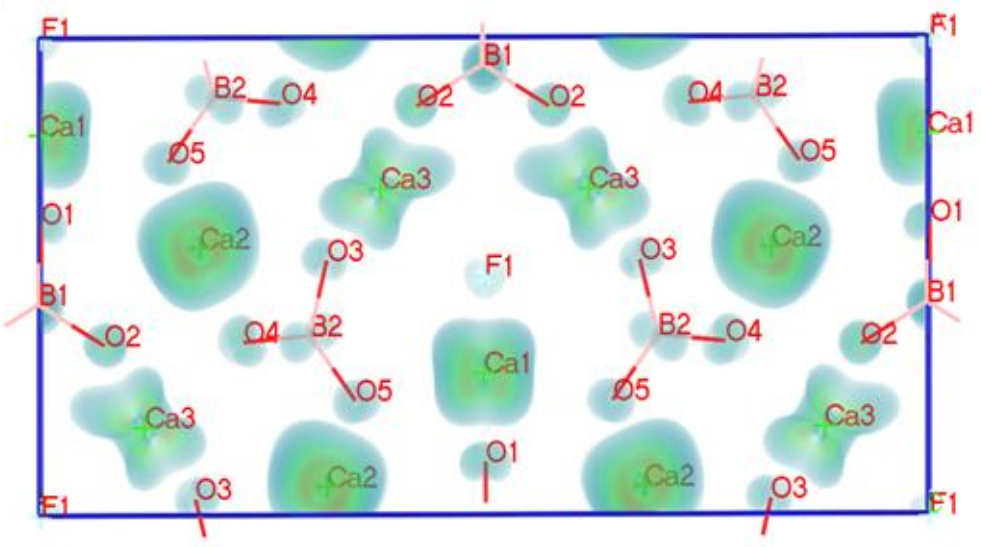

VE unoccupied

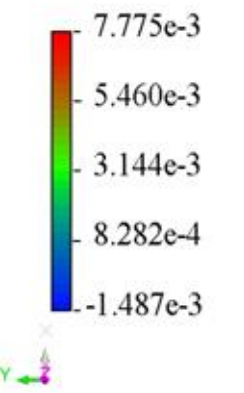

Figure S7. The SHG-density of occupied states and unoccupied states of $\mathrm{Ca}_{5}\left(\mathrm{BO}_{3}\right)_{3} \mathrm{~F}$ in VE process.

\section{References}

1. Huang, Z.; Su, X.; Pan, S. L.; Dong, X.; Han, S.; Yu, H.; Zhang, M.; Yang, Y.; Cui, S.; Yang, Z. H.; Scripta Mater. 2013, 69, 449-452.

2. Yu, H. W.; Wu, H. P.; Pan, S. L.; Yang, Z. H.; Su, X.; Zhang, F. F.; J. Mater. Chem. 2012, 22, 9665-9670.

3. Wu, H. P.; Yu, H. W.; Yang, Z. H.; Hou, X.; Su, X.; Pan, S. L.; Poeppelmeier, K. R.; Rondinelli, J. M.; J. Am. Chem. Soc. 2013, 135, 4215-4218.

4. Zhang, F.; Jing, Q.; Zhang, F.; Pan, S. L.; Yang, Z.; Han, J.; Zhang M.; Han, S.; J. Mater. Chem. C. 2014, 2, 667-674.

5. Chen, G.; Wu, Y.; Fu, P.; J. Cryst. Growth. 2006, 292, 449-453. 